\title{
Meningitis neonatal por Streptococcus pneumoniae atípico: Reporte de un caso y revisión
}

\author{
Verónica Silva B., Felipe Castillo F., Paula O 'Reilly F., Isabel Araya B., Lorena Porte T., \\ M. Teresa Ulloa F., Carmen Varela A. y Juanita Zamorano R.
}

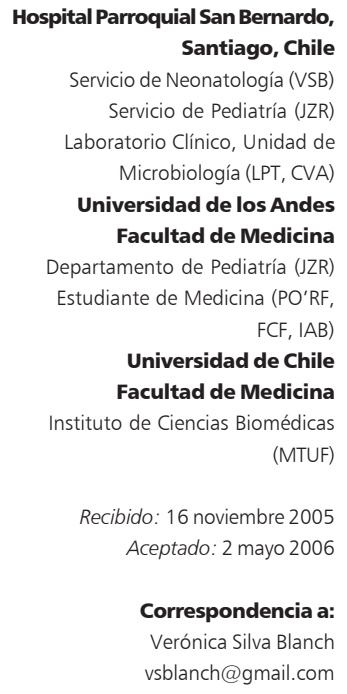

\section{Introducción}

S

treptococcus pneumoniae es una causa infrecuente de sepsis neonatal ( 1 a $11 \%$ ), pero entraña una alta tasa de morbimortalidad tanto para el niño como para la madre. Las manifestaciones clínicas de la infección por este microorganismo son similares a las de la sepsis causada por Streptococcus agalactiae pero $S$. pneumoniae se comporta de manera más agresiva resultando en importante mortalidad $(50 \%)^{1,2}$. Este hecho confirma la importancia de considerar a $S$. pneumoniae dentro de las etiologías infecciosas causantes de sepsis neonatal, principalmente en aquellas de instalación precoz.

Tradicionalmente, esta bacteria se ha identificado en base a su susceptibilidad a optoquina. Sin embargo, la emergencia de cepas de $S$. pneumoniae resistentes a optoquina ("atípicas") hace difícil el diagnóstico sin utilizar varias pruebas diagnósticas, incluyendo las de biología molecular ${ }^{3}$.

Se describe a continuación el caso de un neonato con meningitis causada por $S$. pneumoniae atípico y se realiza una revisión de la literatura científica sobre la evolución clínica de las infecciones asociadas a esta bacteria y el estado actual de su diagnóstico microbiológico.

\section{Caso Clínico}

Recién nacida (RN) de término, segunda hija de madre de 19 años, sana y con embarazo controlado.
Parto vaginal espontáneo, Apgar 9 al minuto y a los 5 minutos, peso de nacimiento: $3.370 \mathrm{~g}$. A las 34 horas de vida, la paciente presentó fiebre hasta $38,8^{\circ} \mathrm{C}$, quejido respiratorio y distensión abdominal, por lo que se decidió su ingreso al Servicio de Neonatología del Hospital Parroquial San Bernardo. Al examen físico destacaban su hipoactividad, mala perfusión periférica, ictericia moderada y el abdomen distendido con leve resistencia muscular.

Los exámenes de laboratorio mostraban leucocitos de 5.300/ $\mathrm{mm}^{3}$ sin desviación a izquierda, PCR: $204 \mathrm{mg} / 1$ y gases sanguíneos con acidosis metabólica. Orina completa y radiografía de tórax normales. La punción lumbar dio un LCR de aspecto turbio. Citoquímico del LCR: glucosa $49 \mathrm{mg} / \mathrm{dl}$, proteínas $266 \mathrm{mg} / \mathrm{dl} \mathrm{y} \mathrm{leucocitos:}$ 950/ $\mathrm{mm}^{3}$ con $70 \%$ PMN, tinción de Gram: cocáceas grampositivas en pares (diplo). Los látex de orina y LCR para detección de antígeno de $S$. agalactiae fueron negativos.

Se inició tratamiento con ampicilina y cefotaxima. Los hemocultivos de ingreso se hicieron positivos a las 7,3 horas de incubación, informándose cocáceas grampositivas en cadena.

La RN persistió febril, hipoactiva y hemodinámicamente estable. Un nuevo LCR obtenido a las 72 horas era de aspecto turbio y tenía 5.050 leucocitos $/ \mathrm{mm}^{3}$ con $100 \%$ PMN. El cultivo de esta muestra fue negativo. Se recibió informe de hemocultivos (2), urocultivo y LCR tomados al ingreso con desarrollo de Streptococcus grupo viridans sensible a penicilina y cefotaxima. El 
laboratorio de microbiología comunicó la identificación como preliminar y sujeta a confirmación.

Al cuarto día de hospitalización y debido a la mala evolución, fue evaluada por infectóloga, suspendiéndose ampicilina y agregando en su reemplazo penicilina $400.000 \mathrm{UI} / \mathrm{kg} /$ día y gentamicina $4 \mathrm{mg} / \mathrm{kg} /$ día como esquema de tratamiento asociado para Streptococcus grupo viridans. La dosis de cefotaxima se aumentó de 100 a $150 \mathrm{mg} / \mathrm{kg} /$ día para optimizar su concentración en el SNC y por la sospecha de absceso cerebral.

Hemocultivos obtenidos intra-tratamiento resultaron negativos, el estudio ecográfico de cerebro y abdomen fueron normales. La ecocardiografía mostró un foramen oval permeable, descartándose la presencia de vegetaciones. La RN evolucionó afebril desde las 48 horas de cambiado el esquema antimicrobiano. Al séptimo día de vida se controló el LCR informándose: 38 leucocitos $/ \mathrm{mm}^{3}$, 93\% PMN, proteínas $164 \mathrm{mg} / \mathrm{dl} \mathrm{y}$ glucosa $46 \mathrm{mg} / \mathrm{dl}$, tinción de Gram sin bacterias y cultivo negativo. No se realizó control ulterior del LCR.

Una vez recibido, al octavo día de tratamiento, el informe microbiológico definitivo de Streptococcus pneumoniae y susceptibilidad corregida según los puntos de corte para meningitis, se completó tratamiento sólo con cefotaxima.

La paciente evolucionó hacia la mejoría recibiendo cefotaxima durante 21 días, duración determinada por la lenta recuperación clínica y de parámetros de laboratorio. Fue dada de alta a los 22 días de vida, en buenas condiciones, no siendo posible realizar seguimiento post alta.

Informe microbiológico. Dos hemocultivos, un urocultivo y el LCR obtenidos al ingresar la paciente resultaron positivos para cocáceas grampositivas en cadena y en diplo. Los hemocultivos se hicieron positivos a las 7,3 horas de incubación (sistema BacT/ Alert ${ }^{\circledR}$, bioMérieux). Se realizó subcultivo en agar sangre de cordero $5 \%$ y agar chocolate (bioMérieux). Las placas se incubaron por 24 horas a $35^{\circ} \mathrm{C}$ en atmósfera con 3 a $5 \%$ de $\mathrm{CO}_{2}$ obteniéndose desarrollo de colonias $\alpha$ hemolíticas umbilicadas. Dado que se obtuvo crecimiento de colonias fenotípicamente similares en todos los cultivos, se realizó la identificación a partir del crecimiento en los hemocultivos.

La prueba de susceptibilidad a optoquina detectó resistencia a ella, por lo que se emitió un informe preliminar de: Streptococcus grupo viridans. El estudio de susceptibilidad para penicilina y cefotaxima se realizó mediante E- test y fue interpretado utilizando los puntos de corte NCCLS/CLSI vigentes ${ }^{4}$ para Streptococcus no pneumoniae resultando sensible para penicilina y cefotaxima (penicilina $0,015 \mu \mathrm{g} / \mathrm{ml}$ y cefotaxima $0,032 \mu \mathrm{g} / \mathrm{ml}$ ). Debido a que el aspecto de la colonia era sugerente de $S$. pneumoniae, se realizó identificación mediante API 20 Strep (bioMérieux) el cual no arrojó resultados concluyentes (S. mitis (33\%), S. oralis $(20 \%)$, S. sanguis $(29 \%))$. La cepa fue enviada al Programa de Microbiología, I.C.B.M., Universidad de Chile, donde uno de los autores (MTUF) realizó prueba de solubilidad en bilis (soluble), detección de antígeno de pared celular de $S$. pneumoniae por inmunocromatografía (BinaxNow $\left.{ }^{\circledR}\right)$ (positivo) y RPC para el gen de neumolisina (positivo), identificándose el microorganismo definitivamente como Streptococcus pneumoniae. La cepa fue enviada al Instituto de Salud Pública cumpliendo la normativa vigente de envío de $S$. pneumoniae invasores al centro de referencia. Se recibió informe de identificación de la cepa como Streptococcus pneumoniae serotipo 5, sensible a penicilina.

Una vez hecho el diagnóstico microbiológico, se corrigió la interpretación de susceptibilidad para cefotaxima según los puntos de corte correspondientes a $S$. pneumoniae de localización meníngea, permaneciendo dentro de la categoría de susceptible tanto a penicilina como a cefotaxima.

\section{Discusión}

Este caso clínico presentó dos situaciones inusuales: meningitis precoz en una RN causada por $S$. pneumoniae y la identificación de una cepa atípica de este microorganismo.

Infecciones causadas por Streptococcus pneumoniae en neonatos. Las infecciones neonatales por $S$. pneumoniae son infrecuentes, reportándose casos esporádicos y pequeñas series de sepsis, neumonía y meningitis. Se estima que entre 1 y $11 \%$ de las sepsis en $\mathrm{RN}$ son causadas por este agente.

Hoffman et al reportaron un total de 29 casos de infección en neonatos, correspondiendo 8 a otitis media aguda y 21 a infecciones bacterianas invasoras (meningitis, 27\%; bacteriemia, 28\%; neumonía, 14\%; infecciones osteoarticulares, 3,4\%). Sólo 3 pacientes presentaron infección sistémica precoz (dentro de las primeras 72 horas) y sólo 1 antes de las 24 horas de vida. El promedio de edad de presentación fue de 18 días y $90 \%$ de los RN fueron de término. Treinta por ciento de los pacientes con infección invasora presentaba leucopenia (glóbulos blancos $<5.000 / \mathrm{mm}^{3}$ ). El serogrupo más frecuente fue el 19, tanto en infecciones invasoras $(30 \%)$ como en otitis media aguda (32\%). Veinte por ciento de las cepas aisladas fueron resistentes a penicilina y una se clasificó como no-susceptible a cefalosporinas de tercera generación. Las cepas resistentes a penicilina se aislaron en los pacientes con el antecedente de tener hermanos y/o hermanos 
que asistían a guardería infantil. La mortalidad en esta serie fue de $14,3 \%$, a diferencia de lo que ocurre en el niño sobre 2 meses de edad, donde la letalidad se concentra en pacientes con patología de base y es de 1,3\%. Las muertes ocurrieron dentro de las 36 horas de presentación ${ }^{2}$.

En el caso descrito destaca la precocidad de los síntomas y la gran alteración de los parámetros citoquímicos del LCR en una RN de término y aparentemente sana.

En la patogenia de la infección invasora del $\mathrm{RN}$ por $S$. pneumoniae se ha atribuido un rol a la transmisión vertical asociada a portación vaginal materna y/o a endometritis ${ }^{2,5,6}$. Mediante estudios de biología molecular se ha comprobado la transmisión clonal de la bacteria desde la madre al niño ${ }^{5}$.

La frecuencia de colonización vaginal por S. pneumoniae es muy baja $(<0,03 \%)$, sugiriendo una alta incidencia de infección invasora en el RN expuesto a este agente durante el parto ${ }^{2}$. Estudios publicados en la década pasada advertían de un posible aumento de la colonización vaginal por este agente, debido al cambio en las prácticas sexuales durante el embarazo (ej: sexo oro-genital) y al desarrollo de mejores técnicas diagnósticas. Mas aún, McDonald y cols plantearon que si la incidencia de sepsis neonatal por $S$. pneumoniae llegara a aumentar al punto de requerirse medidas de prevención específicas, podría considerarse la vacunación materna como una medida útil ${ }^{5}$.

Una preocupación adicional es la posible colonización vaginal de las madres con cepas de $S$. pneumoniae resistentes a penicilina y a cefalosporinas de tercera generación o la selección de cepas resistentes mediante el uso de antimicrobianos para la prevención de infección neonatal por $S$. agalactiae. En la literatura científica internacional se ha descrito que entre $20 \mathrm{y}$ $50 \%$ de las cepas de S. pneumoniae aisladas de RN serían resistentes a penicilina y 4 a $7 \%$ lo serían a cefalosporinas de tercera generación ${ }^{2,7-9}$. En estos casos la profilaxis con penicilina no evitaría la transmisión de cepas resistentes al neonato. Esta es una situación preocupante en muchos países; en Chile, Saldías y cols en su estudio sobre la susceptibilidad a antimicrobianos de $S$. pneumoniae en población infantil y adulta de Santiago entre 1997 y 2003, describieron $10 \%$ de resistencia a penicilina (CIM $>2 \mu \mathrm{g} / \mathrm{ml}$ ) y $20 \%$ de resistencia intermedia (CIM: 0,12-1 $\mu \mathrm{g} / \mathrm{ml}$ ). Para cefotaxima encontraron $2,5 \%$ de resistencia (CIM $>2$ $\mu \mathrm{g} / \mathrm{ml}$ ) y ninguna cepa con $\mathrm{CIM}>4 \mu \mathrm{g} / \mathrm{ml}$. Además, la resistencia a penicilina no cambió significativamente durante el período de estudio, observándose un predominio en la población pediátrica, pero en muestras de sitios no estériles ${ }^{10}$.

Entre los factores de riesgo relacionados a infec- ción por $S$. pneumoniae en el $\mathrm{RN}$ se encuentran la rotura prematura ovular, la prematurez, la neumonía neonatal de presentación precoz $(<72$ horas) y el parto vaginal, siendo este último el único factor de riesgo demostrado ${ }^{11}$. Por esto y, a pesar de la baja frecuencia de $S$. pneumoniae como causa de enfermedad neonatal, resulta importante que se considere a esta bacteria dentro de los probables agentes de sepsis en recién nacidos de madres no portadoras de $S$. agalactiae o que han recibido profilaxis antimicrobiana durante el parto. Si bien en nuestro caso la madre de la $\mathrm{RN}$ no presentó factores de riesgo conocidos para la portación vaginal de $S$. pneumoniae ni se le realizó cultivo vaginal post-parto, la evolución de la RN permite suponer que se trataría de un caso de transmisión vertical intrauterina.

Diagnóstico microbiológico de S. pneumoniae atípico. El segundo hecho inusual en este paciente caso fue la identificación de una cepa de $S$. pneumoniae atípico. Streptococcus pneumoniae es miembro del grupo Streptococcus mitis-Streptococcus oralis perteneciente a los Streptococcus grupo viridans. La diferenciación de $S$. pneumoniae de otros Streptococcus del grupo viridans se ha basado tradicionalmente en características fenotípicas, fundamentalmente en la susceptibilidad a optoquina y/o la solubilidad en bilis $^{3,12}$. Sin embargo, existen reportes de cepas optoquina resistentes que son erróneamente diagnosticadas como Streptococcus $\alpha$ hemolíticos no S. pneumoniae, en los laboratorios en que sólo se utiliza esta prueba presuntiva para la identificación ${ }^{3,13,14}$.

También se han reportado cepas de $S$. pneumoniae, insolubles en bilis, aparentemente asociado a variaciones en la metodología utilizada. Sin embargo, independiente de la técnica usada, esta prueba se describe como el método fenotípico de mayor sensibilidad (>98\%) y especificidad (100\%).

Aún más, otras pruebas fenotípicas tales como los kits comerciales basados en reacciones bioquímicas, entre ellos los sistemas API 20 Strep y Rapid ID 32 Strep ${ }^{\circledR}$ (bioMérieux), generalmente no diferencian $S$. pneumoniae de otros estreptococos del grupo viridans sin el apoyo de las pruebas de optoquina y/o de solubilidad en bilis. Kikuchi et al y Kawamura et al reportaron una baja correlación entre los resultados de estos dos sistemas y entre hibridación ADN-ADN y Rapid ID 32 Strep $^{3}$. Un trabajo anterior de Kawamura et al en que se evaluaron dos tipos de kits de identificación (Rapid ID 32 Strep ${ }^{\circledR}$ y STREPTOGRAM ${ }^{\circledR}$ ) demostró que estos sistemas de identificación presentaban menos de $79 \%$ de exactitud para la identificación de especies pertenecientes al grupo mitis ${ }^{15}$.

La detección de antígenos demuestra alta sensibilidad, pero especificidad limitada, pues se han descrito reacciones cruzadas con Streptococcus grupo viridans 
no pneumococcus. Además, las cepas acapsuladas no pueden ser detectadas por pruebas serológicas utilizadas para diagnóstico y/o tipificación. Este hecho se ha reportado en $2 \%$ de las cepas provenientes de líquidos estériles y hasta en $20 \%$ de los aislados de muestras de secreción conjuntival ${ }^{16}$.

Debido a lo anterior, se ha propuesto el uso de técnicas moleculares que identifiquen genes específicos de S. pneumoniae para un diagnóstico certero. Algunos de los métodos descritos incluyen RPC para los genes de virulencia de $S$. pneumoniae: autolisina (gen $l y t A$ ) y pneumolisina (gen $p l y$ ) y para el antígeno de superficie neumocóccico A ( $p s a A)$. Sin embargo, se ha demostrado la presencia de estos genes en otros miembros del grupo viridans, lo que los inhabilita como herramientas únicas para el diagnóstico de certeza ${ }^{3,16,17}$. Otra técnica genética propuesta es la hibridación de una sonda de ADN con secuencias 16S rARN específicas para $S$. pneumoniae (AccuProbe; GenProbe, San Diego, California), la que, aunque se ha usado como estándar de oro en algunos trabajos, no permite una adecuada discriminación entre especies en todos los $\operatorname{casos}^{3}$.

Si bien los métodos genotípicos entregan nuevas posibilidades para la identificación de esta bacteria, la presencia de genes de virulencia de $S$. pneumoniae en cepas no neumocóccicas de $S$. mitis, probablemente debido a su estrecha relación génica y la transferencia horizontal de genes, dificultan su interpretacion ${ }^{16}$. Es un hecho bien conocido que los miembros del grupo mitis muestran valores de similitud ADN-ADN de 40 a $60 \%$ y algunas especies comparten más de $99 \%$ de homología en la secuencia $16 \mathrm{~S}$ rARN ${ }^{15}$.

Ko et al caracterizaron aislamientos no tipificables de $S$. pneumoniae provenientes de países asiáticos mediante el uso de las pruebas de susceptibilidad a optoquina, solubilidad en bilis, secuenciación multilocus de genes "house keeping" y amplificación de genes de virulencia, digestión $16 \mathrm{~S}$ rADN-RsaI y secuenciación $16 \mathrm{~S}$ rADN, en forma combinada. Se encontraron aislados atípicos que mostraban resultados diferentes a las cepas típicas de $S$. pneumoniae: divergencia en la secuencia de sus genes $r e c P$ y $x p t$, discrepancias en la secuenciación multilocus y en las secuencias $16 \mathrm{~S}$ rADN y del gen lytA y resultados discordantes en las pruebas fenotípicas ${ }^{18}$.

Lo anterior refuerza la necesidad de utilizar una combinación de métodos en el diagnóstico de este tipo de aislamientos, ya que las técnicas existentes por sí solas no permiten un diagnóstico certero. En nuestro medio es fundamental que los laboratorios asistenciales implementen de rutina la prueba de solubilidad en bilis para la correcta identificación de $S$. pneumoniae, frente a cepas de Streptococcus $\alpha$ hemolíticos resistentes o con susceptibilidad disminuida a optoquina. Mas aún, cuando ya hay autores que plantean la emergencia de una nueva especie dentro del grupo viridans llamada Streptococcus pseudopneumoniae, caracterizada por no poseer cápsula, ser resistente a optoquina e insoluble en bilis ${ }^{3}$.

Dada la permanente evolución de los microorganismos y su expresión fenotípica también cabe destacar la importancia de realizar diagnósticos microbiológicos colaborativos que cuenten con los aportes tanto de la microbiología tradicional como de la molecular.

\section{Resumen}

Streptococcus pneumoniae es una causa infrecuente de infección en el recién nacido y se caracteriza por gran capacidad invasora (sepsis, meningitis) y alta mortalidad. Tradicionalmente, esta bacteria se diagnostica en base a su susceptibilidad a optoquina. Sin embargo, la emergencia de cepas de S. pneumoniae resistentes a optoquina (atípicas) dificulta el diagnóstico sin utilizar varias pruebas diagnósticas, incluyendo las de biología molecular. Se describe el caso de una neonata con infección invasora causada por una cepa de $S$. pneumoniae atípico y se discuten los métodos empleados para certificar el diagnóstico de esta especie.

\section{Referencias}

1.- Sallam A, Paes B. Streptococcus pneumoniae: an old bug with significant maternal-newborn implications. Am J Perinatol 2004; 21: 491-5.

2.- Hoffman J A, Mason E O, Schutze G E, Tan T Q, Barson W J, Givner L B, et al. Streptococcus pneumoniae infections in the neonate. Pediatrics 2003; 112:1095-102.

3.- Arbique J C, Poyart C, Trieu-Cuot P,
Quesne G, Carvalho M S, Steigerwalt A G, et al. Accuracy of phenotypic and genotypic testing for identification of Streptococcus pneumoniae and description of

Streptococcus pseudopneumoniae sp. nov. J Clin Microbiol 2004; 42: 4686-96.

4.- Performance Standards for Antimicrobial Susceptibility Testing. CLSI M100-S15. Vol. 25 N¹. 2005.

5. - McDonald L C, Bryant K, Snyder J. Peripartum transmission of penicillin- resistant Streptococcus pneumoniae. J Clin Microbiol 2003; 41: 2258-60.

6. - Hughes B R, Mercer J L, Gosbel L B. Neonatal pneumococcal sepsis in association with fatal maternal pneumococcal sepsis. Aust N Z J Obstet Gynaecol 2001; 41: 457-8.

7.- Pérez López A, Giménez M, Rodrigo C, Alonso A, Prat C, Auxina V. Seven-year review of paediatric bacteraemias diagnosed in a Spanish university hospital. Acta Paediatr 2003; 92: 854-6. 
8.- Bernaola E, Aristegui J, Herranz M, García C, Fernández C. Study of the incidence of invasive pneumococcal disease in neonates and children aged less than 5 years in the Basque country and Navarre. An Esp Pediatr 2002; 57: 301-9.

9.- Schuchat A, Robinson K, Wenger J, Harrison L, Farley M, Reingold A et al. Bacterial meningitis in the United States in 1995. Active Surveillance Team. N Engl J Med 1997; 337: 970-6.

10.- Saldías F, Flores L, Torres M C, García P, Díaz A. Susceptibilidad a antimicrobianos de Streptococcus pneumoniae en población infantil y adulta de Santiago. Periodo 19972003. Rev Méd Chile 2005; 133: 42-9.

11.- Alba C, Ramos J T, Gómez E. Pneumococci: a new microorganism in the newborn. An Esp Pediatr 2001; 54: 390-3.
12.- Lund E, Henrichsen J. Laboratory diagnosis, serology and epidemiology of Streptococcus pneumoniae. Methods Microbiol 1978; 12: 241-63.

13. - Borek A P, Dressel D C, Hussong J, Peterson L R. Evolving clinical problems with Streptococcus pneumoniae: increasing resistance to antimicrobial agents and failure of traditional optochin identification in Chicago, Illinois, between 1993 and 1996. Diagn Microbiol Infect Dis 29: 209-14.

14.- Pikis A, Campos J M, Rodríguez W J, Keith J M. Optochin resistance in Streptococcus pneumoniae: mechanism, significance, and clinical implications. J Infect Dis 2001; 184: 582-90

15.- Kawamura Y, Whiley R, Shu S, Ezaki T, Hardie M. Genetic approaches to the identification of the mitis group within the genus Streptococcus. Microbiology 1999; 145: 2605-13

16.- Verhelst R, Kaijalainen T, De Baere T, Verschraegen G, Claeys G, Van Simaey L, et al. Comparison of five genotypic techniques for identification of optochinresistant pneumococcus-like isolates. J Clin Microbiol 2003; 41: 3521-5.

17.- Dagan R, Shriker O, Hazan I, Leibovitz E, Greenberg D, Schlaeffer F, et al. Prospective study to determine clinical relevance of detection of pneumococcal DNA in sera of children by PCR. J Clin Microbiol 1998; 36: 669-73.

18.- Ko K S, Oh W S, Peck K R, Lee J H, Lee N Y, Song J H. Phenotypic and genotypic discrepancy of Streptococcus pneumoniae strains isolated from asian countries. FEMS Immunol Med Microbiol 2005; 45: 63-70. 